\title{
Factores condicionantes de depresión en trabajadores metalúrgicos
}

\author{
Cecilia Díaz ${ }^{1}$, Augusto Ramírez ${ }^{2}$, Pablo Tejada \\ ${ }^{1}$ Médica Residente UNMSM, ${ }^{2}$ American College of Occupational and Environmental Medicine, \\ ${ }^{3}$ Southern Perú CC.
}

\begin{abstract}
Resumen
OBJETIVOS: Buscar factores determinantes en el desarrollo del cuadro clínico de depresión. MATERIAL Y MÉTODOS: Estudio de 153 trabajadores ferroviarios y operadores de grúa-puente en una empresa minero metalúrgica de cobre, evaluados con la Escala de Hamilton. Se detecta 25 casos probables de depresión, quienes son examinados por el médico psiquiatra. En 13 se confirma el diagnóstico y para fines comparativos constituyen el grupo «casos». El resto de la población, 140, integran el grupo "control". Se compara los grupos estadísticamente por regresión logística. Usamos el Programa SPSS / 6,1 y el intervalo de confianza se calcula para el 95\% de certeza. RESULTADOS: Se encuentra una prevalencia de depresión de 8,49\%. El sentimiento de pérdida actual, disfunción familiar severa, condiciones de trabajo inadecuadas y desempeño laboral presentan razón de probabilidad con valor de p altamente significativo $(59,3 ; 11,4 ; 10,0$ y 11,2 , respectivamente). Edad, estado civil, nivel de instrucción, consumo de alcohol o tabaco, antecedentes personales o familiares de trastorno psiquiátrico y dolencia orgánica crónica no son significativos. CONCLUSIONES: La depresión en la población estudiada está fuertemente asociada a sentimiento de pérdida actual, disfunción familiar severa, desempeño laboral y condiciones de trabajo inadecuadas, que constituyen los factores de riesgo.
\end{abstract}

Palabras clave: Depresión; factores de riesgo; condiciones de trabajo; minería; personal de operación.

\section{Depression in metallurgical workers Summary}

OBJECTIVE: To seek determinant factors in the development of depression clinical picture. MATERIAL AND METHODS: Study by Hamilton's Scale of a copper metallurgical mining company 158 railroad men and gantry-crane men workers. Twenty-five probable cases of depression were referred for psychiatric evaluation. In 13 diagnosis was confirmed and represent the cases group and the remaining 140 men, the control group. Both groups were compared statistically by logistic regression. Confidence intervals were calculated at $95 \%$. RESULTS: Depression prevalence was $8,49 \%$. Feeling of current loss, family severe dysfunction, improper working conditions and occupational performance represented probability reasons with $\mathrm{P}$ value highly meaningful $(59,3 ; 11,4 ; 10,0 ; 11,2$, respectively). Age, civil state, education level, alcohol or tobacco consumption, personal or familiar history of psychiatric disorder, chronic organic illness were not meaningful. CONCLUSIONS: Depression in the studied workers is strongly associated with feeling of current loss, severe familiar dysfunction, occupational performance and inadequate working conditions, constituting risk factors.

Key words: Depression; risk factors; working conditions; mining; operators.

\section{INTRODUCCIÓN}

Correspondencia:

Dr. Augusto V. Ramírez

Las Mandarinas 210. Ap 306

Lima 12, Perú.

E-mail:augustovram@yahoo.es 
La palabra depresión deriva de la raíz latina "premo", que indica peso, carga, tormento, opresión. No existe una definición totalmente aceptada y se ha tratado mas bien de describirla en función de algunos síntomas, como por ejemplo, "estado de ánimo deprimido, disminución del disfrute, apatía y pérdida de interés en el trabajo, sentimiento de minusvalía, insomnio, anorexia, ideación suicida, etc." ${ }^{1}{ }^{1}$. Constituye una categoría psicopatológica que puede adoptar forma de síntoma, síndrome o enfermedad $\left(^{2}\right)$.

El hombre es un ser biopsicosocial y por tanto enfrenta constantes situaciones de adaptación al medio, que en algunos casos conllevan la aparición de un trastorno muy frecuente en la actualidad, la depresión. Ella es considerada ya como una enfermedad propia de nuestra cultura. Crea, además, problemas de Salud Pública, por su alto índice de morbilidad, y el consiguiente impacto económico. Estudios epidemiológicos en América Latina revelan tasas de prevalencia de $9,8 \%$ en República Dominicana, 12\% en Argentina, 15\% en Chile y $11,7 \%$ en nuestro país $\left({ }^{1,2}\right)$.

Si bien los costos directos de la enfermedad depresiva no tratada o tratada inadecuadamente están aumentando en todos los países, los costos indirectos son mayores, pues incluyen aquellos derivados del aumento en la utilización de los servicios de salud para el tratamiento de problemas somáticos que encubren depresión subyacente, hospitalización prolongada, incremento del riesgo de padecer enfermedad física y aún de muerte prematura por suicidio. El medio laboral también es alterado por ausentismo, disminución de la productividad y accidentes laborales. En el fondo, siempre hallamos merma de la calidad de vida, objetivo de todo sistema de salud, afectando así no sólo al paciente sino también al entorno familiar, laboral y social $\left.{ }^{\beta, 4}\right)$.

Desde siempre se ha considerado que el tipo de ocupación tiene efecto en el mantenimiento de un buen estado físico y mental del trabajador, pero no ha sido sino en las dos últimas décadas que se ha podido documentar científicamente. Además, es obvio que la sobrecarga de trabajo o la falta de éste, la lucidez requerida para realizar una tarea, la necesidad de tomar decisiones en forma rápida y oportuna, la fatiga por el esfuerzo físico o las tareas repetitivas, el trabajo por turnos, el excesivo número de horas de trabajo por los mal llamados «sobretiempos», los cambios frecuentes en el contenido del trabajo, la nueva tendencia impuesta por las empresas para que el trabajador desarrolle varias tareas en un mismo puesto de labor, llamada pomposamente "polifuncionabilidad", llevan a una situación de estrés que de por sí puede condicionar depresión $\left(^{3-5}\right)$.

Consideramos también que la mayor parte de enfermos deprimidos no consulta al médico general $\mathrm{y}$ menos al psiquiatra $\mathrm{y}$, si tenemos en cuenta las repercusiones laborales, familiares, psicológicas y sociales que trae consigo la depresión, se entiende que ésta constituye actualmente el gran tema de la medicina en general y de la clínica psiquiátrica en particular $\left({ }^{6-8}\right)$.

Estudios de prevalencia de depresión en atención primaria de salud han dado cifras variadas a nivel nacional y mundial. Creemos que esto se debe principalmente a los diversos métodos y conceptos empleados en su diagnóstico; y, es más, son estudios parciales o limitados; por ello, el porcentaje de enfermedad depresiva que afecta a la población general ha sido motivo de controversia, estimándose cifras que van de 4 a $15 \%$. Estudios en Francia ( ${ }^{9}$ ) sobre factores ocupacionales como condicionantes del desorden ansioso o depresivo en trabajadores de una compañía de gas y electricidad, encuentran que los que trabajan en ocupaciones peligrosas están mejor protegidos contra estos desórdenes, mientras que los supervisores son más propensos a desarrollarlos. Otro de Estados Unidos sobre diferencias ocupacionales respecto a depresión y salud global indica que tanto en profesionales como en ocupaciones con mando, se dan los calificativos más saludables, mientras que las ocupaciones que incluyen a operadores de máquinas y transportistas tienen los calificativos más pobres $\left({ }^{10}\right)$.

Cornejo cita a la Organización Mundial de la Salud señalando que $4 \%$ de la población general requiere tratamiento por depresión. En su estudio 
hecho a pacientes ambulatorios del Hospital Hipólito Unanue en Tacna encuentra que de las variables propuestas como factores de riesgo asociados en ambos sexos, son influyentes y estadísticamente significativas: zona de procedencia, antecedentes psiquiátricos familiares y consumo de tabaco; fuertemente influyentes: acontecimiento estresante reciente, grado de estabilidad familiar, relación con el cónyuge, grupo familiar, viudez y separación $\left({ }^{11}\right)$. Un estudio nacional realizado por Warthon, Novara y Sotillo con la finalidad de estandarizar las escalas para medir depresión más usadas entre nosotros, encuentran que la prevalencia del trastorno depresivo es de $65,7 \%$ según la escala de Zung y de $80 \%$ según la de Hamilton, sin diferencia significativa entre ambos métodos $\left({ }^{12}\right)$.

Los estudios para averiguar la influencia de la ocupación sobre la depresión son pocos, sobre todo los que usan criterios diagnósticos estandarizados para abordar el área del trastorno mental en el lugar de trabajo vs. factores de riesgo ocupacional asociados a ellos. En general, los resultados demuestran que es mayor la frecuencia de depresión en los niveles extremos de las categorías ocupacionales, mientras que la calidad del trabajo realizado estaría correlacionada directamente con el nivel ocupacional $\left({ }^{13}\right)$.

Respecto al riesgo empírico de que un familiar del enfermo deprimido padezca la misma enfermedad, existe entre 10 a $15 \%$ de posibilidades de que los hijos de un padre deprimido presenten un cuadro semejante. Si el afectado es un hermano, el riesgo sería de la misma magnitud. Si los dos progenitores fueran deprimidos graves, sus descendientes afrontarían $50 \%$ de posibilidades de padecer el mismo síndrome. Con familiares más lejanos, como tíos, abuelos y primos, el riesgo disminuye a 3 ó 4\%. Aquellos con familiares cercanos que están deprimidos, comparados con quienes no los tienen, la posibilidad de llegar a estar deprimido es casi dos veces mayor. El riesgo de depresión en varones es de 5 a $12 \%$, pero cuando el varón sufre una enfermedad crónica puede elevarse hasta $33 \%\left({ }^{14,15}\right)$.
Referente al consumo de alcohol, no se ha encontrado relación clara entre éste y el estado depresivo. En varones se ha podido observar un mejor nivel afectivo entre los que consumen bebidas alcohólicas ocasionalmente, que entre los abstemios y bebedores cotidianos. Los estudios epidemiológicos establecen que la comorbilidad de estos dos trastornos va de 15 a $65 \%$ y que la asociación entre depresión y dependencia a alcohol es mucho más fuerte cuando hay abuso de alcohol. Aunque es frecuente que el cuadro alcohólico produzca depresión secundaria, a veces ella enmascara una depresión primaria. Tampoco hay una relación clara entre depresión, consumo de tabaco $\mathrm{u}$ otras sustancias $\left({ }^{6,8}\right)$.

Es relevante señalar que, en determinados casos, como sucede algunas veces con el trabajador que vive en un campamento minero y el cónyuge no vive en el lugar, aunque pueda estar presente en forma esporádica o bien asiste allí sólo para mantener una situación de prestigio personal y evitar la desaprobación social. Esta situación de convivencia indiferente o agresiva sería más dañina que las anteriores $\left({ }^{16,17}\right)$.

Los problemas de tipo laboral son también factores psicodinámicos importantes. Hay estudios relevantes en poblaciones trabajadoras de países industrializados $(3,4,9,10)$.

El diagnóstico de la depresión es eminentemente clínico y para los fines de este estudio nos hemos basado en los criterios diagnósticos de la Décima Revisión de la Clasificación Internacional de Enfermedades, CIE $10\left({ }^{18}\right)$. Existen diferentes escalas para valorar la depresión, entre ellas, la de Valoración de Depresión de Hamilton y la de Autovaloración de Depresión de Zung han sido las más usadas en los últimos años:

La de Hamilton es ampliamente usada y muy efectiva para vigilar el estado depresivo en el tiempo y para evaluar la efectividad de un instrumento antidepresivo. Entre sus ventajas está el manejo rápido, válido y confiable cuando es dirigida por personal capacitado. Su limitación 
estaría en que da énfasis marcado a síntomas biológicos, neurovegetativos y síntomas depresivos sicóticos. Si se la usa, debe acompañársela de una evaluación adicional de cambios que se sabe coadyuvan a los estados depresivos, como estado de ánimo, afecto y estado cognitivo, por cuanto no es instrumento diagnóstico de depresión $\left({ }^{12,15,19}\right)$.

Mientras tanto, la escala de Zung es una escala de autovaloración, con la limitación que no se adapta a cambios y por tanto no podría ser usada para evaluar el curso del tratamiento en el paciente deprimido; puede más bien ser usada para la identificación clínica de poblaciones con depresión $\left({ }^{12,14}\right)$.

Para averiguar hasta qué punto la depresión podría ser considerada como factor de riesgo en accidentes laborales, se investigó este problema, para contribuir así a reducir los accidentes. Para ello hicimos un estudio observacional de casos y controles, aplicando la Escala de Hamilton para valorar la depresión, y otros cuestionarios auxiliares para verificar otros factores que pudiesen estar asociados.

Se encontró que la tasa de prevalencia de depresión era de $8,49 \%$ a predominio de episodio depresivo leve $y$, por los resultados de la evaluación estadística, concluimos que los principales factores de riesgo asociados al desarrollo de depresión en el lugar de trabajo son: disfunción familiar severa, trabajo específico como ferroviario y condiciones de trabajo inadecuadas.

\section{MATERIAL Y MÉTODOS}

Los objetivos del estudio fueron determinar la prevalencia de depresión en los trabajadores ferroviarios y grueros de una empresa minero metalúrgica y establecer, entre estos mismos trabajadores, un sector que reuniese características de normalidad necesarias para ser manejado como "grupo control" y realizar la comparación con el "grupo casos" en quienes, obviamente, los síntomas de depresión eran ya manifiestos. Finalmente, se deseaba comparar estadísticamente ambos grupos.
El estudio fue diseñado como observacional retrospectivo de casos y controles.

La población a estudiar estuvo constituida por todos los trabajadores $(n=158)$ que laboraban como operadores de grúa puente en Fundición y Refinería, a quienes denominamos "grueros", y por todos los maquinistas, conductores, brequeros o "linieros" a quienes denominamos en general "ferroviarios", pertenecientes a la Unidad Metalúrgica de una Empresa Minera de la Gran minería peruana, todos de sexo masculino $\left({ }^{20}\right)$.

Se excluyó a los trabajadores con trastorno afectivo bipolar y aquellos que recibían medicación antidepresiva o antihipertensiva. Por tanto, se excluyó a 5 trabajadores y el grupo a estudiar quedó conformado por 153.

Para el estudio usamos la Escala de Hamilton estandarizada por el Instituto Nacional de Salud Mental Honorio Delgado - Hideyo Noguchi $\left({ }^{2}\right)$, la cual hace una valoración descendente y emplea 17 síntomas depresivos típicos para explorar la depresión en forma objetiva. Adicionalmente, se usó otros criterios relevantes desde el punto de vista del diagnóstico diferencial, para la valoración psicopatológica. De acuerdo a esta escala consideramos los siguientes valores:

- Ausencia de síntomas depresivos (0 a 6 puntos).

- Síntomas de intensidad leve (7 a 14 puntos).

- Síntomas de intensidad moderada (15 a 18 puntos).

Si bien era claro para los investigadores que la escala de Hamilton se aplica más para evaluar el grado de severidad de depresión en pacientes ya diagnosticados, se incluyó como instrumento para este estudio por cuanto fue respaldada por una ficha de factores asociados a depresión. Se descartó la posibilidad de aplicar la escala de autovaloración de Zung, para evitar la negación de síntomas por parte de algunos trabajadores, ante el temor de que este estudio fuese un medio de selección de personal por la empresa

Para evaluar otros factores de riesgo asociados a depresión, como disfunción familiar, sentimientos 
de pérdida, remotos o actuales, además de usoabuso de alcohol, usamos el Cuestionario de Funcionamiento Familiar de Smilkstein $\left({ }^{21}\right)$.

Para valorar el riesgo factor ocupacional usamos la Escala de Chevalier ( $\left.{ }^{9}\right)$, adecuándola a nuestra realidad. Evaluamos los cambios recientes o inminentes en el lugar de trabajo, la exigencia física y psicológica producidas por el trabajo y la percepción del trabajador respecto a su trabajo.

Se hizo una entrevista personal a los 158 trabajadores de las secciones ferrocarril y grueros dirigida a obtener datos para la Escala de Hamilton y para las fichas de Registro de Factores de Riesgo Asociados. Para completar los datos, se revisó la historia médica ocupacional y la historia clínica del Hospital de la empresa. La Escala de Hamilton y la Ficha de Factores Asociados de cada uno de los trabajadores fueron evaluadas por el médico psiquiatra. Todos los trabajadores que, según la escala de Hamilton, presentaban síntomas depresivos y aquellos en los que se consideró necesario, en total 25 , fueron citados nuevamente para entrevista con el psiquiatra quien llegó a establecer el diagnóstico clínico de depresión en 13 trabajadores. Finalmente la población estudiada quedó reducida a 153 trabajadores, ya que dos fueron excluidos por estar recibiendo medicación antihipertensiva y tres no acudieron a la entrevista. Con el resultado de la evaluación psiquiátrica, se formó dos grupos: uno de casos (13 trabajadores con depresión) y el otro de controles (140 trabajadores sin depresión). Se evaluó antecedentes de exposición a un número definido de supuestos factores de riesgo y se hizo la comparación estadística de los grupos.

Los datos obtenidos fueron sometidos a análisis multivariable por el método de regresión logística $\left.{ }^{(22}\right)$ con el programa SPSS/6,1. Establecimos Intervalos de confianza a nivel de $95 \%$ de certeza.

\section{RESULTADOS}

Los resultados del estudio los mostramos en las Tablas 1 a 7.
La Tabla 1 muestra la población estudiada, distribuida por enfermedad, ocupación y prevalencia total. Hallamos que $8,49 \%$ del universo presentaba cuadro depresivo, a predominio del grupo ferroviario $(7,2 \%)$ y sólo $1,3 \%$ de los operadores de grúa.

Luego se presenta a población distribuida por grupos de edad por número y frecuencia porcentual en casos y controles (Tabla 2), el valor de las variables de los estimadores cuantitativos estudiados y muestra media, mediana y desviación estándar de los grupos (Tabla 3) y la frecuencia de las variables de antecedentes de exposición por número y por ciento en ambos grupos (Tabla 4).

En la Tabla 5 está la comparación del valor de los factores de exposición estudiados y el cálculo de $\mathrm{X}^{2}$ con el correspondiente valor de $\mathrm{p}$, la Tabla 6 muestra el resultado de la comparación por regresión logística de las variables seleccionadas y, por último, en la Tabla 7 se presenta el valor de las variables determinantes de depresión aplicando el método de remoción escalonada, con el respectivo valor de $\mathrm{p}$, error estándar, intervalo de confianza y razón de probabilidad.

Tabla 1.- Distribución por enfermedad y ocupación.

n: 153

Sexo: Masculino

\begin{tabular}{cccc}
\hline Depresión & Grueros & Ferroviarios & Total \\
\hline Sí & 2 & 11 & 13 \\
No & 60 & 80 & 140 \\
Total & 62 & 91 & 153 \\
\hline
\end{tabular}

Tasa de prevalencia: $8,5 \%$.

\section{DISCUSIÓN}

Para investigar el problema depresión en este grupo de trabajadores minero metalúrgicos, nos planteamos la siguiente interrogante: ¿son la edad, estado civil, nivel de instrucción, funcionamiento familiar, hábitos nocivos, antecedentes personales y familiares de trastorno psiquiátrico, presencia de enfermedad orgánica crónica, condiciones de trabajo o sentimiento de pérdida, factores de 
Tabla 2.- Distribución de casos y controles por grupos de edad.

\begin{tabular}{ccc}
\hline $\begin{array}{c}\text { Grupo } \\
\text { (Años) }\end{array}$ & $\begin{array}{c}\text { Casos } \\
\text { n (f \%) }\end{array}$ & $\begin{array}{c}\text { Controles } \\
\text { n (f \%) }\end{array}$ \\
\hline $25-29$ & 0 & $3(2,1)$ \\
$30-34$ & 0 & $3(2,1)$ \\
$35-39$ & 0 & $10(7,1)$ \\
$40-44$ & $2(15,4)$ & $29(20,7)$ \\
$45-49$ & $4(30,8)$ & $42(30,0)$ \\
$50-54$ & $1(7,7)$ & $30(21,4)$ \\
$55-59$ & $2(15,4)$ & $12(8,6)$ \\
$60-64$ & $4(30,8)$ & $11(7,9)$ \\
Total & 13 & 140 \\
Valores extremos & $40-64$ & $25-64$ \\
\hline
\end{tabular}

$n(f \%)=$ Número $($ frecuencia por cien $)$.

riesgo para que los trabajadores a estudiar desarrollen el cuadro clínico de depresión?.

Luego de estudiar a la población de trabajadores en cuestión, analizamos los resultados y encontramos que la tasa de prevalencia de depresión en la población estudiada fue $8,49 \%$. Para nuestro medio, este valor se encuentra dentro de las cifras estimadas para la población general, que fluctúan entre 4 y $15 \%$, y es un tanto mayor que el encontrado por Chevalier en un país desarrollado en trabajadores varones, quien da una prevalencia de $7,6 \%\left({ }^{9,11}\right)$.

La mayor frecuencia de depresión ha sido hallada entre los 50 y 60 años $\left({ }^{12}\right)$. En nuestro estudio, el 53,8\% del grupo casos tenía edad igual o mayor a 50 años, comparado con $37,8 \%$ del grupo control. No hemos encontrado que la edad sea un factor riesgo asociado a depresión $(\mathrm{OR}=$ 1,7; IC: $0,1-25,4 ; p=0,69$ ). Esto puede ser porque la depresión sería debida fundamentalmente a cambios psico-sociales a los que se van enfrentando las personas a esa edad, siendo más importantes las condiciones del medio circundante que la edad misma. En la evaluación del estado civil, $76,5 \%$ de los casos y $94,9 \%$ de los controles eran casados o "convivientes" y la frecuencia de aquellos considerados en riesgo para hacer depresión -como viudos o personas solitarias $\left({ }^{13,16,17}\right)$ - fue escaso; por tanto, tampoco encontramos que el estado civil es un factor de riesgo asociado a depresión $(\mathrm{OR}=0,7$; IC: 0,0 $18,9 ; \mathrm{p}=0,81)$. Esto se podría explicar porque, si bien el estado civil de casado o «conviviente» denota una situación en la cual la persona no se encuentra sola, ello no siempre es así entre nuestros trabajadores.

Para evaluar nivel de instrucción y depresión, agrupamos a los trabajadores en tres niveles: primaria, secundaria y técnica o superior (no hay

Tabla 3.- Valor de las variables del estimador cuantitativo.

\begin{tabular}{|c|c|c|c|c|c|c|}
\hline \multirow[t]{2}{*}{ Variables } & \multicolumn{3}{|c|}{ Casos $n=13$} & \multicolumn{3}{|c|}{ Controles $n=140$} \\
\hline & $\mathrm{X}$ & $\mathrm{Md}$ & DE & $\mathrm{X}$ & $\mathrm{Md}$ & $\mathrm{DE}$ \\
\hline Edad & 52,9 & 51,7 & 8,76 & 48,0 & 47,5 & 7,50 \\
\hline Funcionamiento familiar & 4,9 & 5,0 & 2,53 & 6,9 & 7,0 & 1,53 \\
\hline Condiciones de trabajo & 8,7 & 9,0 & 1,10 & 8,3 & 8,2 & 1,33 \\
\hline $\mathrm{N}^{\circ}$ cigarillos/semana & 20,5 & 20,5 & 0,70 & 10,3 & 10,0 & 9,80 \\
\hline
\end{tabular}

$X=$ Media $\quad$ Md = Mediana $\quad D E=$ Desviación estándar

$\left(^{1}\right)$ Total de fumadores en el grupo control: 49, Consumo de cigarrillos en este grupo: valores extremos: 0,5 y 35 . Previo al cómputo se eliminó dos valores, 50 y 70, por ser extremos (outliners); para ello se empleó el procedimiento de la desviación extrema estudantizada (DEE) 
Tabla 4.- Frecuencia de las variables de antecedentes de exposición.

\begin{tabular}{|c|c|c|}
\hline Variable & $\begin{array}{l}\text { Casos } \\
(13) \\
\mathrm{n}(\mathrm{f} \%)\end{array}$ & $\begin{array}{c}\text { Controles } \\
\quad(140) \\
\mathrm{n}(\mathrm{f} \%)\end{array}$ \\
\hline Edad > 50 & $7(53,8)$ & $53(37,8)$ \\
\hline $\begin{array}{l}\text { Estado civil: } \\
\text { - Soltero } \\
\text { - Casado } \\
\text { - Viudo } \\
\text { - Separado } \\
\text { - Divorciado } \\
\text { - Conviviente }\end{array}$ & $\begin{array}{rr} & 0 \\
7 & (53,8) \\
1 & (7,7) \\
1 & (7,7) \\
1 & (7,7) \\
3(23,0)\end{array}$ & $\begin{array}{rr}2 & (1,4) \\
123 & (87,8) \\
0 \\
3 & (2,1) \\
2 & (1,4) \\
10 & (7,1)\end{array}$ \\
\hline $\begin{array}{l}\text { Escolaridad: } \\
\text { - Primaria incompleta } \\
\text { - Primaria completa } \\
\text { - Secundaria incompleta } \\
\text { - Secundaria completa } \\
\text { - Técnica } \\
\text { - Superior }\end{array}$ & $\begin{array}{rr} & 0 \\
3 & (23,0) \\
2 & (15,4) \\
8 & (61,5) \\
0 \\
0\end{array}$ & $\begin{array}{r}6(4,3) \\
17(12,1) \\
25(17,9) \\
79(56,4) \\
12(8,5) \\
1(0,7)\end{array}$ \\
\hline $\begin{array}{l}\text { Funcionamiento familiar: } \\
\text { - Bueno } \\
\text { - Disfunción leve } \\
\text { - Disfunción severa }\end{array}$ & $\begin{array}{ll}4 & (30,7) \\
5 & (38,5) \\
4 & (30,7)\end{array}$ & $\begin{array}{r}95(67,8) \\
41(29,3) \\
4(2,9)\end{array}$ \\
\hline $\begin{array}{l}\text { Consumo de alcohol: } \\
\text { - Abstemio } \\
\text { - Bebedor excesivo } \\
\text { - Bebedor moderado } \\
\text { Consumo de tabaco. }\end{array}$ & $\begin{array}{ll}6 & (46,1) \\
2 & (15,4) \\
5 & (38,5) \\
2 & (15,4)\end{array}$ & $\begin{array}{r}30(21,4) \\
10(7,1) \\
100(71,4) \\
51(36,4)\end{array}$ \\
\hline $\begin{array}{l}\text { Antecedente personal de trast } \\
\text { - Depresión } \\
- \text { Otro }^{1}\end{array}$ & $\begin{array}{r}\text { rno psiqui } \\
1(7,7) \\
6(46,1)\end{array}$ & $\begin{aligned} \text { rico: } & \\
14 & (10,0) \\
31 & (22,1)\end{aligned}$ \\
\hline $\begin{array}{l}\text { Antecedente familiar de trasto } \\
\text { - Depresión } \\
\text { - } \text { Otro }^{2}\end{array}$ & $\begin{array}{r}\text { rno psiquia } \\
0 \\
1(7,7)\end{array}$ & $\begin{array}{l}\text { rico: } \\
\qquad \begin{array}{rr} \\
1(0,7) \\
4(2,9)\end{array}\end{array}$ \\
\hline $\begin{array}{l}\text { Enfermedad orgánica crónica } \\
\text { Condiciones de trabajo } \\
\text { inadecuadas: }\end{array}$ & $10(76,9)$ & $77(55,0)$ \\
\hline $\begin{array}{l}\text { Sentimiento de pérdida: } \\
\text { - Pasada } \\
\text { - Actual }\end{array}$ & $\begin{array}{r}5(38,9) \\
10(76,9)\end{array}$ & $\begin{array}{r}30(21,4) \\
11(7,9)\end{array}$ \\
\hline $\begin{array}{l}\text { Ocupación: } \\
\text { - Ferroviario } \\
\text { - Gruero }\end{array}$ & $\begin{array}{rr}11 & (84,6) \\
2 & (15,4)\end{array}$ & $\begin{array}{ll}80 & (57,1) \\
60 & (42,9)\end{array}$ \\
\hline
\end{tabular}

$n(f \%)=$ Número $($ frecuencia porcien $)$

(1) Síndrome ansioso, alcoholismo crónico, neurosis obsesiva, trastorno del sueño, reacción conversiva (hombro doloroso, cefalea tensional).

(2) Alcoholismo crónico, síndrome ansioso, psicosis de guerra, esquizofrenia.
Tabla 5.- Comparación del valor de los factores de exposición.

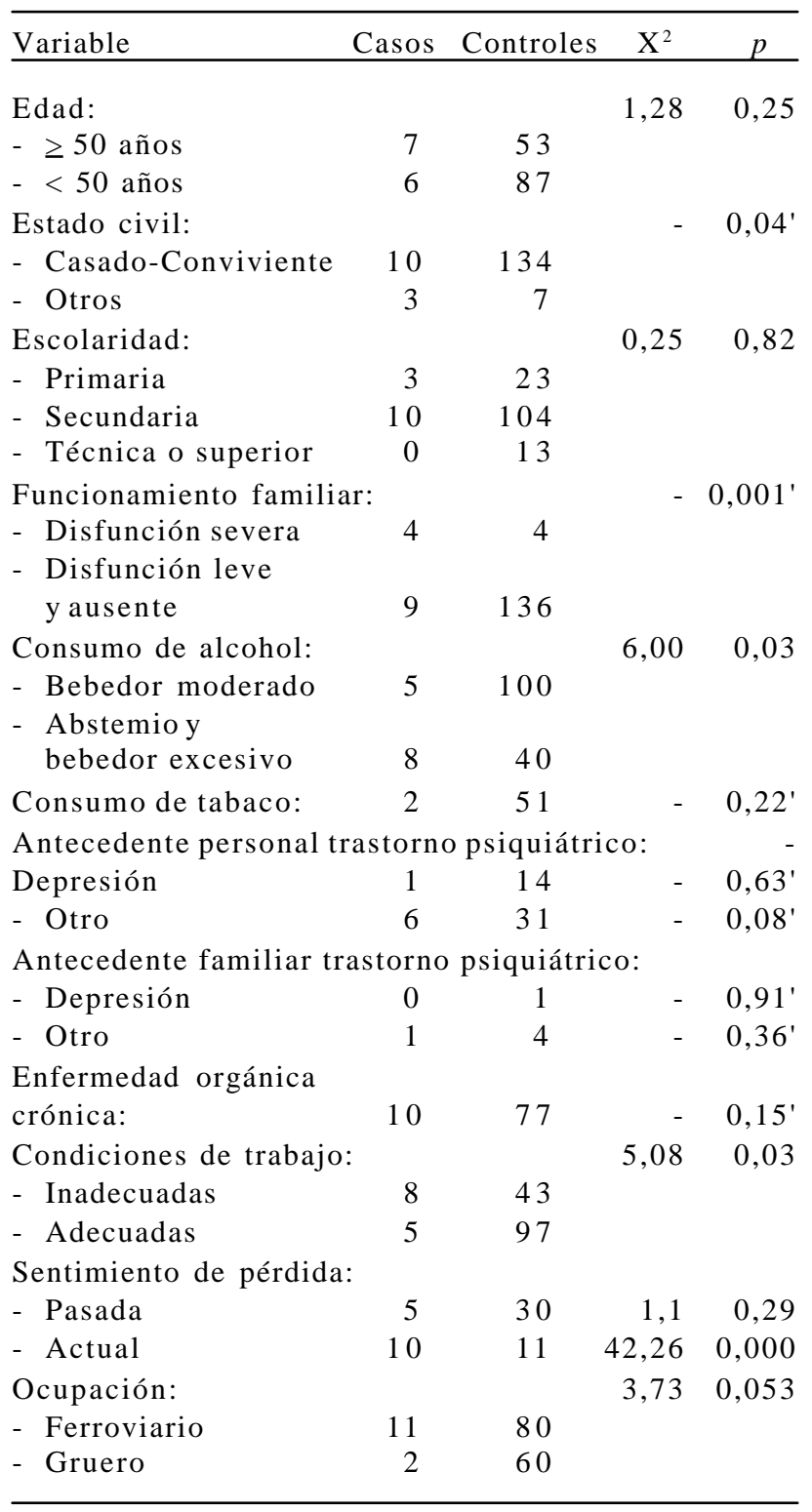

Nota: El valor de p fue calculado con la prueba exacta de Fisher.

analfabetos en la población estudiada). Luego del análisis estadístico, ninguna de estas categorías de instrucción constituye factor de riesgo. Tal vez aquí ha influido el que la mayoría de población tiene nivel secundario $(76,9 \%$ en casos, $74,3 \%$ en controles). Estos porcentajes están en un valor 
Tabla 6.- Valor de las variables de seleccionadas comparadas por regresión logística.

\begin{tabular}{lrrrrrr}
\hline Variable & $\mathrm{b}$ & $\mathrm{SE}$ & \multicolumn{1}{c}{$\mathrm{x}^{2}$} & $p$ & $\mathrm{OR}$ & $\mathrm{IC}$ \\
\hline Edad (FR): & 0,5492 & 1,3705 & 0,16 & 0,69 & 1,7 & $0,1-25,4$ \\
Estado civil (FR): & $-0,4112$ & 1,7091 & 0,06 & 0,81 & 0,7 & $0,0-18,9$ \\
Educación: & & & & & & \\
- Primaria & $-1,3323$ & 3,2323 & 0,17 & 0,68 & 0,3 & $0,0-148,9$ \\
- Secundaria & 0,1741 & 2,7875 & 0,00 & 0,95 & 1,2 & $0,0-280,8$ \\
- Técnica o superior & $-5,9035$ & 39,5532 & 0,02 & 0,88 & 0,0 & $0,0-1,2$ \\
Disfunción familiar severa: & 2,2235 & 1,5512 & 2,05 & 0,15 & 9,2 & $0,4-193,2$ \\
- Alcohol (FR): & 0,7968 & 1,2373 & 0,41 & 0,52 & 2,2 & $0,2-25,1$ \\
- Tabaco (FR): & $-0,7534$ & 1,3540 & 0,31 & 0,58 & 0,5 & $0,0-6,7$ \\
Antecedente personal trastorno psiquiátrico: & & & & & & \\
- Depresión & $-1,7888$ & 2,6247 & 0,46 & 0,50 & 0,2 & $0,0-28,7$ \\
- Otro & $-0,0283$ & 1,4210 & 0,00 & 0,98 & 1,0 & $0,1-15,8$ \\
Antecedente familiar trastorno psiquiátrico: & & & & & & $0,0-\propto$ \\
- Depresión & $-4,9807$ & 164,2726 & 0,00 & 0,98 & 0,0 & $0,0-1,9$ \\
- Otro & 0,9186 & 1,8594 & 0,24 & 0,62 & 2,5 & $0,1-95,9$ \\
Enfermedad orgánica crónica (FR): & $-0,1355$ & 1,2996 & 0,01 & 0,92 & 0,9 & $0,1-11,2$ \\
Condiciones de trabajo (FR): & 2,3819 & 1,2382 & 3,70 & 0,05 & 10,8 & $1,0-122,6$ \\
Ocupación: & 2,7038 & 1,4530 & 3,46 & 0,06 & 14,9 & $0,9-257,7$ \\
Sentimiento de pérdida pasada: & 0,3057 & 1,1207 & 0,07 & 0,79 & 1,4 & $0,2-12,2$ \\
Sentimiento de pérdida actual: & 4,5290 & 1,2418 & 13,30 & 0,00 & 92,7 & $8,1-1056,7$ \\
\hline
\end{tabular}

FR: Factor de riesgo

$S E=$ Error estándar

$O R=$ Odds ratio $($ Razón de probabilidad $)$

$$
\begin{aligned}
& b=\text { Valor del coeficiente de regresión de la variable } \\
& p=\text { Valor de } p \\
& I C=\text { Intervalo de confianza del } O R
\end{aligned}
$$

intermedio respecto otros estudios $\left({ }^{16,17}\right)$. Tengamos en cuenta, además, que nuestros trabajadores reciben capacitación constante relacionada a la labor que desempeñan, lo que evitaría el sentimiento de incapacidad y consiguiente estrés que podría llevar a desarrollar depresión.

Los hábitos nocivos alcohol y tabaco, en nuestro estudio no constituyeron factor de riesgo $(\mathrm{OR}=2,2 ; \mathrm{IC}: 0,2-25,1 ; p=0,52$ y $\mathrm{OR}=0,5$; IC: $0,0-6,7 ; p=0,58)$. Encontramos que 64,5\% de casos y $28,6 \%$ de controles estaba en situación de riesgo (abstemios y bebedores excesivos). En cuanto al tabaco, $15,5 \%$ de casos y $36,4 \%$ de controles consumía un promedio de 20 cigarrillos por semana. Estos resultados son importantes comparados con el estudio de Vela $\left({ }^{17}\right)$, quien encuentra consumo de tabaco similar pero no así el de alcohol, pues 4,5 y $25,5 \%$ eran bebedores patológicos y excesivos, respectivamente; lo cual en cierto modo nos satisface, porque en nuestra población no encontramos bebedores patológicos y los excesivos constituyeron $22,5 \%$ de casos.

Los antecedentes personales de depresión u otro trastorno psiquiátrico tampoco fueron factores de riesgo asociado $(\mathrm{OR}=0,2 ; \mathrm{IC}: 0,0$ 28,$7 ; p=0,50$ y $\mathrm{OR}=1,0 ; \mathrm{IC}: 0,1-15,8 ; p=$ 0,98). Para fines comparativos y en el afán de ser objetivos, tengamos en cuenta que este dato lo obtuvimos de la revisión de la historia clínica y ya que la depresión es un trastorno muchas veces no diagnosticado. La prevalencia real de este antecedente podría haber estado disminuida. Además, considerando que se trabajó con una población supuesta sana, no era de esperar trastorno psiquiátrico alguno como esquizofrenia o demencia senil. Por otro lado, los antecedentes familiares de depresión y otros trastornos psiquiátricos tampoco fueron factor de riesgo (OR 
Tabla 7.- Valor de las variables determinantes de depresión seleccionadas por análisis de remoción escalonada *.

\begin{tabular}{|c|c|c|c|c|c|c|c|}
\hline Variable & $\mathrm{a}$ & $\mathrm{b}$ & SE & $X^{2}$ & $p$ & O R & IC $95 \%$ \\
\hline \multicolumn{8}{|c|}{$-7,0928$} \\
\hline Disfunción familiar severa & & 2,4382 & 1,1995 & 4,13 & 0,04 & 11,4 & $1,1-120,2$ \\
\hline Condiciones de trabajo inadecuadas & & 2,3042 & 0,9691 & 5,65 & 0,01 & 10,0 & $1,5-66,9$ \\
\hline Ocupación ferroviario & & 2,4183 & 1,1152 & 4,70 & 0,03 & 11,2 & $1,3-99,9$ \\
\hline Sentimiento de pérdida actual & & 4,0825 & 0,9817 & 17,29 & 0,00 & 59,3 & $8,7-406,1$ \\
\hline
\end{tabular}

$a=$ Valor de la variable " $y$ " cuando $X=0$

$b=$ Valor del coeficiente de regresion de la variable

$S E=$ Error estándar

$p=$ Valor de $p$

$O R=$ Odds ratio $($ Razón de probabilidad $)$

$I C=$ Intervalo de confianza del OR

* Este análisis consiste en incluir en la ecuación todas las variables seleccionadas. Luego, el modelo identifica aquellas que aportan menos a su explicación y las remueve sucesivamente (remoción escalonada), hasta dejar sólo aquellas variables cuya influencia es significativa para el efecto. La secuencia de remoción fue: antecedente personal de otro trastorno psiquiátrico, educación secundaria, antecedente familiar de depresión, enfermedad orgánica crónica, sentimiento de pérdida pasada, estado civil como factor, antecedente familiar de otro trastorno psiquiátrico, edad como factor, educación técnica o superior, consumo de alcohol como factor, educación primaria, consumo de tabaco y antecedente personal de depresión $\left({ }^{17}\right)$.

$=0,0$; IC: $0,0-\mu ; p=0,98$ y OR =2,5; IC: 0,1 $95,9, p=0,62$ ), quizá también por el criterio cuasi selectivo ya mencionado que empleamos para registrar este dato o simplemente porque éste no fue referido por el trabajador.

Ser portador de enfermedad crónica orgánica no constituyó factor de riesgo (OR=0,9; IC: 0,1 $11,2 ; p=0,92)$. La frecuencia encontrada en casos y controles fue de 76,9 y 55\%, respectivamente. Esto podría ser explicado porque los trabajadores y sus familiares son objeto de controles médicos frecuentes; y, es más, cuando enferman, la empresa les proporciona atención médica gratuita y oportuna, por lo que cualquier enfermedad no es percibida con la misma magnitud con la que lo harían personas que no gozan de estas ventajas.

En cuanto a funcionamiento familiar, sí encontramos que la disfunción severa es un factor de riesgo asociado a depresión, presente en $30,7 \%$ de casos y en $2,9 \%$ de los controles $(\mathrm{OR}=$ 11,4; IC $1,1-120,2 ; p=0,04)$. Esto concuerda con los resultados de Chevalier $\left.{ }^{9}\right)$, quien si bien no evaluó funcionamiento familiar en sí encontró que $15,6 \%$ de trabajadores con depresión tenía problemas con sus cónyuges contra un $5,4 \%$ de los controles $(p<0,01)$, y que en $13,6 \%$ de casos había dificultad importante a causa de los hijos, frente a un 5,1\% de controles $(p<0,01)$. También, en el estudio de Vela, la prevalencia de familia desintegrada fue alta $\left({ }^{17}\right)$. Esta situación se explicaría porque nuestros trabajadores viven en un "campamento minero" y los hijos, concluida la secundaria, emigran a la ciudad en busca de estudios superiores, con la concomitante migración del cónyuge del trabajador, dejando a éste en situación de soledad, que se ha observado es importante en el desarrollo de la depresión $\left({ }^{14}\right)$.

Condiciones de trabajo inadecuadas también fueron un factor de riesgo asociado a la depresión para esta población $(\mathrm{OR}=10,0$; IC: 1,5 - 66,9; $p$ $=0,01$ ), con frecuencia de $61,5 \%$ en el grupo casos y $30,7 \%$ en el de control. Esto podría deberse a una suerte de precaria estabilidad en el trabajo, condicionada por cambios en el modelo económico nacional, que ha obligado a las empresas a aplicar programas de reducción de costos que el trabajador percibe como disminución 
del monto de ingreso al que estuvo acostumbrado. Además, en el subconsciente del trabajador está presente algo que podríamos llamar "pérdida colectiva del trabajo" por la tendencia cada vez mayor de la empresa a utilizar services en lugar de trabajadores "estables" o de "planilla". Esto corrobora lo encontrado por Chevalier $\left(^{9}\right)$, quien sugiere que algunos eventos ocupacionales tales como cambios importantes en el contenido, metodología u organización del trabajo pueden causar o precipitar desorden ansioso / depresivo, cuya influencia es de la misma magnitud que aquellos factores no relacionados al trabajo.

E1 $38,5 \%$ de casos y $30 \%$ de controles presentaron sentimiento de pérdida pasada, que no constituye factor de riesgo asociado $(\mathrm{OR}=1,4$; IC: $0,2-12,2 ; p=0,79)$. En cambio, para sentimiento de pérdida actual (OR $=59,3$; IC 8,7 - 406,1; $p=0,00)$, presente en 76,9\% de casos y $7,9 \%$ de controles, sí hemos encontramos que era un factor importante asociado a depresión; esto podría sustentarse principalmente en la percepción de inestabilidad laboral referida en el párrafo anterior.

En este mismo sentido, consideramos importante evaluar la ocupación como factor de riesgo. Encontramos que la de ferroviario sí lo fue $(\mathrm{OR}=11,2 ; \mathrm{IC}: 1,3-99,9 ; p=0,03)$. Respecto a ocupación y depresión, son muy pocos los estudios publicados, pero es claro que el tipo de trabajo de la persona puede contribuir a su presentación. En este grupo de trabajadores podrían haber influido los cambios recientes o inminentes ya descritos, con el mismo peso que las exigencias físicas y psicológicas producidas por el tipo de trabajo, motivo por el cual el servicio de Salud Ocupacional de la Empresa ya lo había considerado como una población en riesgo.

En conclusión, la tasa de prevalencia de depresión en el grupo estudiado fue de $8,49 \%$, a predominio de episodio depresivo leve $6,53 \%$; mientras tanto, el episodio depresivo moderado llegó a 1,96\%. Por la evaluación estadística, concluimos que los principales factores de riesgo asociados a su desarrollo fueron disfunción familiar severa, trabajo como ferroviario o gruero y condiciones inadecuadas de trabajo.

Recomendamos hacer seguimiento especializado a todos los trabajadores diagnosticados de depresión. Y, como corolario, los resultados que presentamos deberían ser orientadores para abordar la investigación científica en Salud Ocupacional, con un enfoque integral que trascienda su estado actual de ser sólo sustento para la cumplir con la obligación legal que tienen las empresas mineras de implementar programas de Higiene y Seguridad Industrial.

\section{BIBLIOGRAFÍA}

1. American Psychiatric Association. Diagnosis and Statistical Manual of Mental Disorders, $4{ }^{\mathrm{Ed}}$ Ed. Washington DC, USA: American Psychiatric Association. 1994.

2. Bertein DA, Nietzel BM. Introducción a la Psicología Clínica. México: Ed. Mc Graw-Hill; 1982.

3. Barry L, Wegman D. Occupational Health Recognizing and Preventing Work Relates Disease. Boston / Toronto: Little Brown and Company, 1998.

4. Grosh J, Murphy L. Occupational differences in depression and global health: Results from a national sample of US workers. J O E M 1998; 40(2): 153-63.

5. Zenz K, Ed. Occupational Medicine. $3^{\text {rd }}$ Edition. St. Louis, USA: Ed Mosby - Year Book Inc. 1994.

6. Paterniti S, Niedhammer I, Lang T, Consoli SM. Psychosocial factors at work and depressive symptoms. B J Psychiatric 2002; 181(2): 111-7.

7. Vidal G, Alarcón R. Psiquiatría. Buenos Aires, Argentina: Editorial Médica Panamericana S. A. 1986.

8. Toro G. Fundamentos de Medicina: Psiquiatría. Medellín, Colombia Corporación Para Investigaciones Biológicas. 1997.

9. Chevalier A. Occupational factors of anxiety and depressive disorders in the French National Electricity and Gas Company. J O E M 1996; 38(11): 1098-107.

10. Lehmer M, Bentley A. Treating work stress: An alternative to workers compensation. J O E M 1997; 39(1): 63-7.

11. Cornejo AM. Depresión en pacientes ambulatorios de Medicina Interna del Hospital Hipólito Unanue en Tacna en el mes de julio de 1995. Tesis Br en Med Humana, Facultad de Medicina U. N. de San Agustín, Arequipa; 1997.

12. Warthon D, Novara J, Sotillo C. Estandarización y correlación de las escalas de Beck, Hamilton y Zung para la depresión en la población de Lima Metropolitana. Instituto Nacional de Salud Mental Honorio Delgado - Hideyo Noguchi. Lima; 1985.

13. Díaz VC. Factores de riesgo asociados a depresión en trabajadores manuales de una compañía minera. Tesis Br. en 
Medicina Humana, Facultad de Medicina, U. N. San Agustín, Arequipa; 1999.

14. Kaplan H, Sadock B. Comprehensive Textbook of Psychiatry. Vol. VI. Williams and Wilkins; 1995.

15. Sagástegui A,Ed.Ansiedad, demencia y otras comorbilidades en depresión. Diagnóstico 1998; 37(5).

16. Cruz L. Prevalencia del síndrome depresivo en el pueblo joven Campo de Marte. Arequipa. Tesis Br. en Enfermería. Fac. de Enfermería U N de San Agustín, Arequipa; 1984.

17. Vela QA. Prevalencia de depresión en pacientes ambulatorios del Hospital Cuajone. Tesis Br. en Medicina Humana, Fac Medicina U N de San Agustín, Arequipa; 1985.

18. Organización Panamericana de la Salud. Clasificación Estadística Internacional de Enfermedades y Problemas Relacionados con la Salud. CIE 10. Décima Revisión, USA: Ofic. Sanitaria Panamericana, 1995.

19. Gibbons RD. Exactly what does the Hamilton Depression Rating Scale measure? J Psychiatry 1993; 27(3): 259-73.

21. Bristol Myers Squibb Net Health Internet. Who's At risk for Depression? Why?. Image Depression.com The Good News About Depression; 1998.

20. Ministerio de Energía y Minas. Perú. Decreto Supremo 046- 2001 EM. Lima; 2001.

22. Schlesselman JJ.Case Control Studies: Design, Conduct, and Analysis. Oxford University Press; 1982. 